\title{
Perforation of the oesophagus by a swallowed foreign body presenting as a mediastinal and pulmonary mass
}

\author{
P J RADFORD, F C WELLS
}

From the Surgical Unit, Papworth Hospital, Cambridge

Perforation of the oesophagus from any cause, including a swallowed foreign body, usually presents with rapid onset of severe pain, followed by the development of fever, perhaps subcutaneous emphysema, and then septicaemia and shock. Very rarely presentation may be delayed and we report an extremely unusual case where the diagnosis was made four months after perforation, when a chronic mediastinal and pulmonary inflammatory mass containing a swallowed bone was found at thoracotomy.

\section{Case report}

A 51 year old man was admitted to Papworth Hospital in July 1986 for open exploration of an upper mediastinal mass to establish a histological diagnosis. He gave a four month history of severe interscapular pain, associated with intermittent anterior chest pain, general malaise, weight loss, and sleep disturbance. His symptoms had begun with a sudden onset of interscapular pain immediately after a meal of pork during which he had swallowed a bone. There was no history of temporary bolus obstruction or of dysphagia either initially or subsequently during the evolution of his illness. His past medical history was unremarkable. He smoked 40 cigarettes per day and worked as a shop floor manager. His mother had died from oesophageal carcinoma.

Examination revealed an ill looking man with erythema ab igne between the scapulae, but no other specific physical abnormality. During the preceding four months he had been investigated at the referring hospital, and found to have a persistently raised erythrocyte sedimentation rate of 80-85 $\mathrm{mm}$ in the first hour. His white cell count was raised $(12 \times$ $\left.10^{9} / 1\right)$, with neutrophilia; but there was no other haematological abnormality. He had increased alkaline phosphatase (479 (normal range 98-279) IU/1) and $\gamma$ glutamyltransferase $(275$ (normal range < 50 ) IU/l). Blood cultures and autoantibody profile were normal and there was no Bence-Jones protein in his urine. Serum protein electrophoresis showed non-specific changes with mildly raised concentrations of $\mathrm{C} 3$ complement and immunoglobulins. Chest radiographs and electrocardiographs were normal on several occasions.

As a result of his persisting symptoms an isotope bone scan was performed which showed increased uptake around the third thoracic vertebra. Computed tomography of this area disclosed a large posterior mediastinal mass engulfing the third and fourth thoracic vertebral bodies (figure). A needle biopsy of this area showed a few inflammatory cells but not Address for reprint requests: Mr F C Wells, Surgical Unit, Papworth Hospital, Papworth Everard, Cambridge.

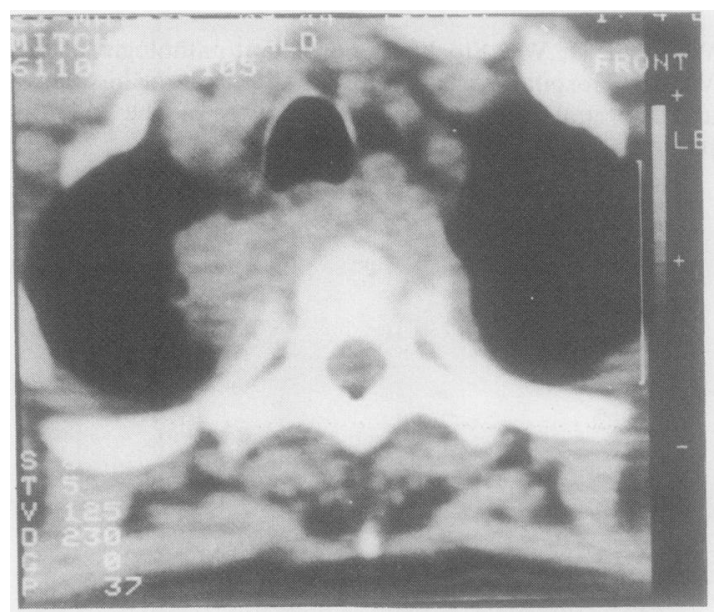

Preoperative computed tomography scan showing a large posteriorly situated mass anterior and to the right of the midline behind the trachea.

the expected malignancy, so he was referred for an open exploration.

Bronchoscopy carried out before surgery showed nothing abnormal. At right lateral thoracotomy the upper lobe of the right lung was adherent to the mediastinum and vertebrae. This part of the lung was firm and indurated. No discrete mediastinal mass was found but the pleurae and mediastinal $O$ tissues were thickened and indurated. Biopsy specimens were taken and the indurated portion of lung removed. Oesophagus and trachea were normal. Histological examination of the mediastinal biopsy specimen showed chronic inflam- N matory changes throughout. Examination of the resected portion of lung disclosed pieces of dead bone surrounded by $\mathrm{N}$ abundant foreign body giant cells. The appearances were $\mathrm{N}$ those of chronic mediastinitis following oesophageal perfora- $\sigma$ tion by a swallowed bone that had migrated to the adjacent lung.

The patient made a full recovery, with resolution of the changes shown by computed tomography when this was? repeated two months later.

\section{Discussion}

Perforation of the oesophagus by a foreign body is an uncommon cause of oesophageal perforation. A recent large 
series' reported only $14 \%$ of 127 cases of perforated oesophagus, seen over 47 years, as being due to this cause.

The risk of perforation after impaction of a foreign body in the oesophagus is well documented in published reports at around $1-2 \%$ of cases. For example, in a large series of 2394 cases $^{2}$ only 25 patients developed perforation. Of these, three died and the other 22 developed mediastinal abscess requiring surgical drainage, illustrating the seriousness of this condition. The mechanism of perforation by an impacted foreign body is thought to be a combination of local inflammation of the wall of the oesophagus and direct pressure necrosis.

Migration of a foreign body to tissues outside the oesophagus is rare, with only four published reports, in which migration to the subcutaneous tissues of the neck, ${ }^{3}$ the thyroid gland, ${ }^{4}$ the pulmonary ligament, ${ }^{5}$ and a major blood vessel $^{6}$ were reported. Migration into the lung itself has not to our knowledge been previously reported.

The mode of presentation of our case was also extremely unusual in terms of its chronicity. Oesophageal perforation usually presents clinically in a dramatic fashion. Chest radiographs are usually abnormal with cervical or mediastinal emphysema, pneumothorax, or pleural fluid. Within the first 24 hours surgical exploration carries the best prognosis, mortality being $8 \%$. After 24 hours from perforation the prognosis deteriorates rapidly. Both surgical and conservative regimens carry a high mortality, ' with up to $100 \%$ mortality for conservative treatment and up to $80 \%$ mor- tality for surgical treatment after 48 hours. We assume that local containment of infection in our case allowed progression to local chronic inflammation.

This case shows that oesophageal perforation may occasionally present in an atypical chronic fashion and that foreign bodies may migrate to tissues adjacent to the oesophagus. This case underlines the importance of seeking a histological diagnosis in cases of mediastinal mass, even to the extent of open exploration should this be necessary, lest an erroneous diagnosis should be made and inappropriate treatment given.

\section{References}

1 Bladergroen MR, Lowe JE, Posthlethwait RW. Diagnosis and recommended management of esophageal perforation and rupture. Ann Thorac Surg 1986;42:235-9.

2 Nandi P, Ong GB. Foreign body in the oesophagus. Review of 2394 cases. Br J Surg 1978;65:5-9.

3 Stieder JW. Surgery of the esophagus. N Engl J Med 1950;243: 445-54.

4 Jemerin AF, Aronoff JS. Foreign body in the thyroid following perforation of the oesophagus. Surgery 1949;25:52-9.

5 Barber GB, Peppercorn MA, Ehrlich C, Thurer R. Esophageal foreign body perforation: report of an unusual case and review of the literature. Am J Gastroenterol 1984;79:509-11.

6 McLaughlin RT, Morris JD, Haight C. The morbid nature of the migrating foreign body in the esophagus. $J$ Thorac Cardiovasc Surg 1966;55:188-92. 